\title{
A non-randomized chlinical trial The effect of pelvic floor exercises at home and electrical stimulation plus biofeedback in hospital in management of stress urinary incontinence of primiparas in short-term postpartum period
}

Fei Tang

Maternal and Child Hospital of Hubei Province, Tongji Medical College, Huazhong University of Science and Technology

Yun Zhao ( $\nabla$ zhao020060@163.com)

Maternal and Child Health Hospital of Hubei Province, Affiliated Hospital of Tongji Medical College, Huazhong University of Science and Technology

https://orcid.org/0000-0002-2323-2926

\section{Guo-qiang Sun}

Maternal and Child Health Hospital of Hubei Province, Tongji Medical College, Huazhong University of Science and Technology

Ying Lin

Maternal and Child Health Hospital of Hubei Province, Tongji Medical College, Huazhong University of Science and Technology

Wan Tang

Maternal and Child Health Hospital of Hubei Province, Tongji Medical College, Huazhong University of Science and Technology

Research article

Keywords: stress urinary incontinence, pelvic floor muscle training, electrical stimulation, biofeedback

Posted Date: November 1st, 2019

DOI: https://doi.org/10.21203/rs.2.16565/v1

License: () (1) This work is licensed under a Creative Commons Attribution 4.0 International License. Read Full License 


\begin{abstract}
Background To investigate the effect of pelvic floor exercises at home and electrical stimulation plus biofeedback in hospital in management of stress urinary incontinence (SUI) primiparas in short-term postpartum period.
\end{abstract}

Methods A total of 150 SUI primiparas at postpartum 6-8 weeks were interviewed. According to participants' willingness and necessary coordination, the subjects were divided into 2 groups including the treatment group $(n=69)$ and the control group $(n=81)$. The treatment group received at-home pelvic floor muscle training(PFMT) every day and in-hospital electrical stimulation plus biofeedback therapy twice a week for consecutive 5 weeks (from 6-8 postpartum weeks to 11-13 postpartum weeks). The control group was not given pelvic floor exercise at home or in hospital. The PFM strength in the two groups was measured by specially trained personnel using digital palpation (Modified Oxford scale:0-5 grade) and surface electromyography(sEMG) in 6-8 postpartum weeks and in 11-13 postpartum weeks. For statistical analysis, t -test,Chi-square analysis and Wilcoxon test were carried out.

Results The recovery rate of SUI in the treatment group was $63.8 \%(44 / 69)$, which was higher than that in the control group $(12.3 \%, 10 / 81)(p<0.05)$. In the treatment group, the PFM strength was higher after PFMT than that before PFMT no matter measured by digital palpation or by $s E M G(p<0.05)$; but in the control group, it had no change at postpartum 6-8 weeks and at postpartum about 12 weeks $(p>0.05)$.

Conclusion: The combination treatment of at-home PFM training and in-hospital electrical stimulation plus biofeedback PFM therapy is an effective method to manage SUI and improve PFM strength in short-time postpartum.

\title{
Background
}

Pelvic floor disorders (PFDs) are common in adult women, especially in women during pregnancy and postpartum period ${ }^{[1]}$. PFDs of women mainly refer to stress urinary incontinence (SUI), pelvic organ prolapsed (POP), sexual dysfunction and fecal incontinence ${ }^{\text {[2] }}$. PDFs can negatively affect women's selfperception of their body image and life quality ${ }^{[3]}$. Many studies showed that the damage of pelvic floor muscles (PFMs) was closely related to SUI in shorttime postpartum period ${ }^{[4-6]}$. Hence, early implementation of preventive strategies on pelvic floor muscle training (PFMT) is essential. PFMT can be carried out at home or in outpatient, which includes PFM exercise, electrical stimulation and biofeedback training. During PFMT, a lot of women are unable to contract the muscles correctly, especially at the start of the training program. Studies ${ }^{[7]}$ showed that inadequate activation of muscle groups and incorrect breathing patterns were the most common mistakes when PFM exercising. It has been proved that supervised training protocol is more effective than oral instruction alone. Digital palpation and surface Electromyography (sEMG) recordings are often applied to teach PFM contraction and evaluate the effect of PFMT [8-9].

This study investigates the therapeutic effect of at-home PFM training combined with in-hospital electrical stimulation plus biofeedback therapy on Chinese primipara with SUI in the short-term postpartum period. The scale of PFM strength was examined using digital palpation and sEMG, and questionnaire survey method was carried out by specially trained personnel before and after the PFM training.

\section{Methods Ethical approval}

The study protocol was approved by the Ethics Committee of Maternal and Child Health Hospital of Hubei Province (201301) and all included women signed written informed consent.

\section{Selection of patients and study design}

Women who had delivered in the obstetric department and visited in the pelvic floor rehabilitation center in postpartum 6-8 weeks period at the Maternal and Child Health Hospital of Hubei Province, Tongji Medical College, Huazhong University of Science and Technology from January 2016 to January 2017 were selected as research objects. Inclusion criteria included Chinese singleton primiparas, age ranging from 20 to 35 years old, clean lochia, no obstetric complications, and no history of accepting PFMT during pregnancy and at postpartum 6-8 weeks period. Exclusion criteria included age less than 20 years old or more than 35 years old, non-Chinese nationality, multiple pregnancies, vaginitis and urinary tract infection, and mental incapacity. A total of 217 SUI primiparas at postpartum 6-8 weeks were interviewed (SUI was diagnosed by questionnaire). The PFM strength was measured by specially trained personnel using digital palpation (Modified Oxford scale:0-5 grade) and sEMG. According to participants' willingness to PFMT and through some necessary coordination, the subjects were divided into 2 groups including the treatment group and the control group. In the treatment group, at-home PFMT was given every day under the guidance of specialized medical personnel and in hospital electrical stimulation plus biofeedback therapy was given twice a week for 5 weeks from 6-8 postpartum weeks to 11-13 postpartum weeks. The control group was not given pelvic floor exercise at home or in hospital. At postpartum 11-13 weeks, the PFM strength in the two groups was measured again using digital palpation and sEMG, and SUI was diagnosed by questionnaire again. All the investigation and examination results were recorded truthfully, including patient characteristics, history, and pregnancy delivery data.

\section{The diagnosis of SUI}

Diagnostic criteria ${ }^{[10]}$. SUI was diagnosed from the following four aspects. $\bigotimes$ to test is urine routine was normal, 邓to test whether there was nervous system disease, \to test whether there were other diseases of urinary system, 凶to diagnose whether the patient cannot help leaking urine during coughing, sneezing, laughing, changing position or upon sudden increase of abdominal pressure. 


\section{Determination of PFM strength using digital palpation}

Digital palpation ${ }^{[11]}$. PFM was determined by specialized medical personnel using digital palpation. First of all, women were told to empty the bladder and took lithotomy position. Then the specialized personnel put the index and middle fingers $2-3 \mathrm{~cm}$ into the vagina and identify the levator ani muscle, separate the two fingers and fall on two sides of levator ani muscle, meanwhile, they put the other hand on the abdomen to make sure the abdominal muscle was relaxed. PFM contraction without any movement of the pelvis or visible contraction of the glutei, hip, or abdominal muscles was emphasized. According to contraction strength and retraction capability, the PFM strength was divided into 0-5 grades and 6 classes. The scale of pelvic floor muscle strength was described by Modified Oxford scale. (Table 1)..

\section{Determination of pelvic floor muscle strength by sEMG}

PFM sEMG was recorded using a small diameter vaginal probe with two metal sensors (Noraxon U.S. A. Inc.) with a preamplifier (band pass filter 20Hz-

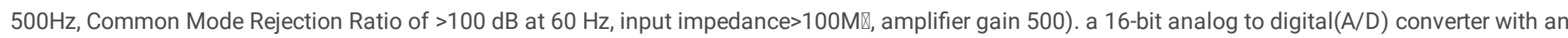
$500 \mathrm{~Hz}$ anti-aliasing filter was used. The probe was inserted using a small amount of antiallergic lubricant positioned laterally in the vagina. After cleansing the skin with an alcohol swab, round self-adhesive electrodes (silver chloride) were applied to the skin over the examined muscle. On the basis of guaranteeing the patients not make visible contractions of these muscles, the next two bipolar self-adhesive electrodes were located on the right side along muscle fibers of the rectus abdominis, hip adductor muscle and gluteus maximus.

Here below is the MVC procedure, and SEMG signal was recorded during each trial (Fig 1) ${ }^{[12]:}$

--a 10-second baseline sEMG recording.

--5 repeated short (quick flick) contraction with a 5 -second pause between each contraction, average mean (MVC) (mean amplitude value of the active sEMG portions, which is calculated when the amplitude exceeds the threshold level predetermined as $50 \%$ between minimum and maximum amplitudes in each particular trial) is analyzed.

--5 repetitions of 10 -second voluntary contractions with 10 seconds of rest in between. Parameters measured: average mean (MVC).

--a sustained 60-second contraction. The magnitude of external forces or loading is not sufficient to initially estimate forces generated by the pelvic floor muscle. The only way to ensure correct performance of the task is to use the maximal contraction and provide verbal encourage to maintain the contraction. Therefore, the instruction "pull up and in, and squeeze around the probe as strongly as you can until you hear the command now relax" is given during the trial. The average mean of contraction of the sEMG signal is analyzed (MVC).

--a 10 s relaxation (resting tone) immediately after the 60 s contraction.

\section{PFM training at home ${ }^{[4]}$}

When SUI women in 6-8 weeks postpartum period was diagnosed, the specialists in our department of pelvic floor center would teach them how to contract their PFM by demonstrating vaginal palpation. At first, the women were told to empty the bladder and took lithotomy position, then the specialist placed her index finger into the vaginal and told the women to lift and squeeze around the finger. For Kegel exercise at home, a sustained 5-second contraction with 10 second pause was performed as a cycle, 10 cycles as a repetition, and 10 repetitions were the amount of training for one day with supine position or upright position. Such training was regularly carried out for 5 weeks at home. The PFMT at home was monitored by the specialists and nurses in our department through WeChat (the most popular online chat platform in China designed by Tencent Company).

\section{Electrical stimulation and biofeedback ${ }^{[13,14]}$}

PFM enforcement program in hospital was carried out by the help of biofeedback and electrical stimulation, and 10 implementations as a complete course. The PFM enforcement program was implemented 30 minutes every time, twice a week, and totally performed for 5 weeks. MyoTrac infiniti (SA9800) device (Thought Technology Inc., Montreal, Canada) was adopted in this experiment. The biostimulation feedback system is based on biofeedback technology, bioformatics principles and bioengineering technology. Safe and effective electrical stimulation combined with biofeedback technology was carried out through vaginal sensor, which could record PFM activity and pelvic floor muscle contraction strength and administer the strength of electrostimulation. The direct vaginal probe consisted of 3 reference electrodes on the iliac and two sides hypogastric skin guiding to prevent the abdominal or gluteus muscles contraction. A separate probe was used for each primiparas to prevent cross-infection. With the help of computer graphics, various parameters of the device were adjusted to make each woman feel the active and passive contractions of PFM and without feeling any pain or discomfort. The parameters of electrical stimulation included the pulse width of $20-40$ us and low voltage of $40-80 \mathrm{~Hz}$. The electrostimulation included rapid contraction exercise of PFM (stimulation and interval time is $2 \mathrm{~s}: 2 \mathrm{~s}$ ) and continuous contraction exercise of PFM (stimulation and interval time is 5-8 s:10 s). The electrical stimulation therapy lasted for $20 \mathrm{~min}$, followed by 10-min biostimulation feedback therapy. Each treatment of electrical stimulation plus biostimulation lasted 30 min, which was conducted two times per week, with a total of 10 times as a full course of treatment.

Testing procedure

Page 3/9 
The experiment consisted of two phases, including at-home PFM training which was carried out every day and in-hospital electrical stimulation combined with biofeedback therapy which was performed twice a week for consecutive 5 weeks

\section{The statistical method}

All the statistical analyses were carried out using the Statistical Package of Social Sciences software (SPSS Version 13.0 Inc., Chicago, IL, USA). The values and variables were reported as mean \pm standard deviation. Student's test was performed to compare the variables in Gaussian distribution. Chi-square test was used to evaluate the categorical variables. Wilcoxon test was used to evaluate the difference of the PFM function at postpartum 6-8 weeks and at postpartum 11-13 weeks. The difference was considered statistically significant at $p<0.05$. A two-sample test was performed to determine whether the mean in the treatment group was different from the mean in the control group. $a=0.05 \otimes \beta=0.1$ \power $=0.90$, sampling ratin $=1$.

\section{Results}

\section{Demographic data of the treatment group and the control group}

A total of 217 SUI primiparas at postpartum 6-8 weeks were interviewed (SUI was diagnosed by questionnaire), excluding 2 cases with non-Chinese nationality, 3 cases of irregular vaginal bleeding, 12 cases for not cooperative with specially trained personnel and 50 cases for not come back to examine the PFM strength at postpartum 11-13 weeks. There were 69 cases in the treatment group and 81 cases in the control group (See Fig 2: diagram flow). Through statistical comparison, it can be known that the difference was not statistically significant between the treatment group and the control group on maternal age, gestational weight gain, BMI of delivery, gestational age at birth, new baby's weight, and delivery modes (Table 2).

\section{The recovery rate of SUI in the treatment group and in the control group}

2-sided equality test was carried out to calculate sample size, the sample size was 61 for every group, and there were 69 cases in the treatment group and 81 cases in the control group, the sample size was enough. The recovery rate of SUl in the treatment group $(63,8 \%, 44 / 69)$ was higher than that in the control group $(12.3 \%, 10 / 81)$ at $11-13$ postpartum weeks according to questionnaire and Chi-square test $(p \otimes 0.05)($ Tab 3)..

\section{The PFM strength measured by digital palpation and sEMG}

In the treatment group, the PFM strength was weak before accepting PFMT at 6-8 postpartum weeks, and the PFM strength was increased with average 0.94 grade after accepting PFMT for 5 weeks. A comparison of the PFM strength using digital palpation revealed significant differences after accepting PFMT at postpartum 11-13 weeks( $\$ \llbracket 0.05$ ) (Tab 4).. Moreover, the PFM strength was found significantly increased after PFMT according to sEMG. The mean amplitude of the sEMG signal from the PFM quick flick, 10-second voluntary contractions, a sustained 60-second contraction after the PFMT for 5 weeks were higher than those before the PFMT(Tab 5)((p/0.05).

In the control group, the PFM strength at 11-13 postpartum weeks was as weak as that at 6-8 postpartum weeks by digital palpation and sEMG ( $p \otimes 0.05)$ $(\operatorname{Tab} 4,5)$..

For both groups, there was no significant difference in PFM strength after PFMT at 6-8 postpartum weeks ( $p \llbracket 0.05)$. However, the PFM strength was stronger in the treatment group than that in the control group at 11-13 postpartum week $(p[0.05)(\operatorname{Tab} 4,5)$.

\section{Discussion}

SUI is a common PFDs during pregnancy and postpartum ${ }^{[1-3]}$. Lin YH et al ${ }^{[15]}$ investigated that $51.5 \%$ (446/866) had self-reported SUI during pregnancy and $12.5 \%$ (70/560) women accepting vaginal delivery and 7.2\% (22/306) women accepting cesarean delivery had SUI at postpartum 12 months. Zhang G et al

${ }^{[16]}$ found SUI was more likely to manifest as leakage of urine when the abdominal pressure gradually increased with the development of pregnancy weeks. The SUI prevalence was 7.5\% (9/120), 22.5\% (27/120), 43.3\% (52/120), and 5.8\% (7/100), in 34, 36, and 38 gestational weeks, and 6 weeks after delivery, respectively. Our previous research showed that the SUI rate of vaginal delivery was $6.8 \%(187 / 2749){ }^{[4]}$. SUI exerts a negative impact on the quality of life. How to recover from SUI as soon as possible after delivery is the focus of our research. Post-delivery SUI is correlated with the weak strength of PFM ${ }^{[4,5]}$. In our study, PFMT at home and in hospital was carried out for consecutive 5 weeks from 6-8 postpartum weeks to 11-13 postpartum weeks. Results showed that 63.8\% (44/69) women could recover from SUI with accepting PFMT, and 12.3\% (10/81) could recover from SUI without accepting PFMT. This shows that short-time postpartum PFMT is effective in the treatment of SUI.

How to effectively exercise PFM strength? Arnold Kegel ${ }^{[17]}$ is known as the first researcher to use PFMT in the treatment of urogynecology dysfunctions. Soon after his studies, many training protocols have been proposed ${ }^{[18-19]}$. Liu L ${ }^{[6]}$ observed 72 menopausal female patients with SUl, among which 24 patients received electrical stimulation therapy combined with biofeedback for 60 days and displayed a clear improvement in their urinary incontinence, pelvic floor muscle strength, leakage times, frequency of urination, urine dynamics index. Electrical stimulation combined with biological feedback seems to bring about better clinical effects than conventional pelvic floor muscle exercises. Schmitt JJ ${ }^{[20]}$ observed that patients received 4-7 sessions (1 every 2 weeks) including biofeedback plus electrical stimulation to ninety-four PFDs patients with urinary or defecatory dysfunction, pelvic pain, dyspareunia, then had a high rate of self-reported subjective success and satisfaction after PFMT by questionnaire, then PFMT should be considered a nonsurgical treatment option in patient with 
PFDs. Wang QJ ${ }^{[21]}$ evaluated the effect of electrical stimulation combined with biofeedback in the treatment of postpartum pelvic organ prolapse through static and dynamic MRI before and after treatment. Results showed that the thickness of bilateral puorectal muscles was thicker, and levator ani hiatus area, $\mathrm{H}$ line, $\mathrm{M}$ line and levator ani plate angle in strain state decreased at postpartum 12 weeks (after PFMT). Our study found that the combination method of PFMT at home and electrical stimulation plus biofeedback in hospital was effective in management of SUI of primiparas in short-term postpartum period. The PFM strength was increased by average 0.94 grade according digital vaginal palpation and the quick flick and $60 \mathrm{~s}$ sustained contraction were higher according to the results of $S E M G$.

More than one method has been adopted to obtain a comprehensive understanding of PFM function [22-23]. However, it is difficult to evaluate PFM function and there is no gold standard for such evaluation ${ }^{[24]}$. Digital vaginal palpation and sEMG evaluation are two common methods to evaluate the strength of PFM. Yang $X$ et al ${ }^{[8]}$ assessed 1,380 parturients at $6-8$ postpartum weeks by using both vaginal palpation and sEMG. Results showed that the contraction variables of EMG had a significant correlation between them and SEMG was also correlated with SUI. In our study, digital vaginal palpation was used to make a general investigation on the PFM strength based on a larger sample size ${ }^{[4,9]}$. sEMG was used for evaluation of the PFMT effect and clinical research. After accepting PFMT for 5 weeks, the higher PFM strength was found by digital vaginal and sEMG.

\section{Conclusions}

Pregnancy and delivery are two independent risk factors leading to SUI of primipara women in the short-time postpartum period. After accepting at home PFM exercise and in hospital biofeedback plus electrical stimulation of PFM, it can be known that the PFM strength was increased by average 0.94 grade according to digital vaginal palpation and the quick flick, and 60 s sustained contraction was higher according to sEMG evaluation. So, conducting PFM exercise at home and electrical stimulation plus biofeedback therapy in hospital for 5 weeks may be helpful for SUI in short-time postpartum period.

\section{Limitation}

1.There are still limitations in the use of sEMG, the inability to assess a specific muscle activity in isolation, the cross-talk effect, artifact motion, and the moving trend of probes during PFM contractions.

2.Our questionnaire on SUI is too simple. Australian Pelvic Floor Questionnaire includes 15 items to reflect Bladder Function ${ }^{\text {[25] }}$, which should be referenced.

\section{Abbreviations Section}

PFDs: pelvic floor disorders

SUI: stress urinary incontinence

POP: pelvic organ prolapsed

PFMs: pelvic floor muscles

PFMT: pelvic floor muscle training

sEMG: surface electromyography

MVC: Maximal Voluntary Contraction

\section{Declarations}

\section{Ethics approval and consent to participate}

The study protocol was approved by the Ethics Committee of Maternal and Child Health Hospital of Hubei Province (201301) and all included women signed written informed consent.

\section{Consent for publication}

Not applicable

\section{Availability of data and material}

This study obtains the consent from the participants to report individual patient's data (see in Additional file 1)

\section{Competing interests}

The authors declare that they have no competing interests. 


\section{Funding}

This work was supported by the Natural Science Foundation of Hubei Province, China (No: 2019CFA033) and Foundation from Health and Family Planning Commission of Hubei Province (No: WJ2015MB121). No authors received any external funding for the present study.

\section{Authors' contributions}

FT participated in the conception and design of the study, data collection, data analysis and manuscript writing. YZ participated in the conception and design of the study, data collection, data developments and manuscript writing.GS participated in the design of the study, data developments. YL and WT participated in the data collection and data developments. All authors read, edited and approved the final version of manuscript.

\section{Acknowledgement}

The authors are thankful to stuffs of the Center of pelvic floor rehabilitation for providing questionnaire by face to face or telephone call.

\section{References}

1. van Veelen GA, Schweitzer KJ, van der Vaart $\mathrm{CH}$. Ultrasound imaging of the pelvic floor: changes in anatomy during and after first pregnancy. Ultrasound Obstet Gynecol. 2014, 44(4): 476-480

2. Bø K. Pelvic floor muscle training in treatment of female stress urinary incontinence, pelvic organ prolapses and sexual dysfunction. World $\mathrm{J}$ Urol. 2012, 30(4):437-443

3. Jelovsek JE, Barber MD. Women seeking treatment for advanced pelvic organ prolapsed have decreased body image and quality of life. Am J Obstet Gynecol. 2006, 194(5):1455-1461

4. Yun Z, Mei X, Fei T, Wan T, Heng Y, Guo-Qiang S, et al. The effect of water immersion delivery on the strength of pelvic floor muscle and pelvic floor disorders during postpartum period. An experimental study. Medicine, (2017) 96:41

5. Burti JS, Hacad CR, Zambon JP, Polessi EA, Almeida FG. Is there any difference in pelvic floor muscles performance between continent and incontinent women? Neurourol Urodyn. 2015, 34(6):544-548.

6. Liu L, Zhang Y, Gong J, Chen X, Wu H, Zhu W. Effects of Different Treatment Methods on the Clinical and Urodynamic State of Perimenopausal Women with Stress Urinary Incontinence. Iran J Public Health. 2018, 47(8):1090-1097.

7. Lamin E, Parrillo LM, Newman DK, Smith AL Pelvic Floor Muscle Training: Underutilization in the USA.Curr Urol Rep. 2016, 17(2):10

8. Yang X, Zhu L, Li W, Sun X, Huang Q, Tong B, et al. Comparisons of Electromyography and Digital Palpation Measurement of Pelvic Floor Muscle Strength in Postpartum Women with Stress Urinary Incontinence and Asymptomatic Parturients: A Cross-Sectional Study. Gynecol Obstet Invest. 2019 Jul 23:1-7.

9. Yun Z, Li Z, Mei X, Wan T, Hai-yi N, et al.Effect of different delivery modes on the short-term strength of the pelvic floor muscle in Chinese primipara. BMC pregnancy and childbirth. 2018,18:275.

10. Abrams $P$, Cardozo L, Fall M, Griffiths $D$, Rosier $P$, Ulmsten $U$, et al. The standardization of terminology in lower urinary tract function: report from the standardization subcommittee of the International Continence Society. Urology. 2003, 61 (1): 37-49.

11. Chen X, Gong Y, Wu D, Li X, Li H, Tong X, et al. Pre- and postoperative evaluation of pelvic floor muscle function in POP patients using surface electromyography and digital palpation. Neurourol and Urodyn. 2014, 33(4):403-407

12. Hacad CR, Glazer HI, Zambon JP, Burti JS, Almeida FG.Is there any change in pelvic floor electromyography during the first 6 months after radical retropubic prostatectomy? Appl Psychophysiol Biofeedback. 2015, 40(1): 9-15.

13. Lee IS, Choi ES. Pelvic floor muscle exercise by biofeedback and electrical stimulation to reinforce the pelvic floor muscle after normal delivery.aehan Kanho Hakhoe Chi.2006, 36(8):1374-1380.

14. Yang S, Sang W, Feng J, Zhao H, Li X, Li P,et al.

The effect of rehabilitation exercises combined with direct vagina low voltage low frequencyelectric stimulation on pelvic nerve electrophysiology and tissı A randomised controlled trial.J Clin Nurs.2017, 26(23-24): 4537-4547

15. Lin YH, Chang SD, Hsieh WC, Chang YL, Chueh HY, Chao AS, et al. Persistent stress urinary incontinence during pregnancy and one year after delivery; its prevalence, risk factors and impact on quality of life in Taiwanese women: An observational cohort study. Taiwan J Obstet Gynecol. 2018, 57(3): 340-345.

16. Zhang G, Jiang W, Guo Q, Guo Q. Value of UVJ-M in the diagnosis of SUI in late pregnancy and postpartum. Exp Ther Med. 2016, 11(5):1772-1776

17. Kegel AH. Progressive resistance exercise in the functional restoration of theperineal muscles. Am J Obstet Gynecol 1948, 56: 238-249.

18. Burns PA, Pranikoff K, Nochajski TH, Hadley EC, Levy KJ, Ory MG. A com-parison of effectiveness of biofeedback and pelvic floor muscle exercisetreatment of stress incontinence in older-community dwelling women. J Gerontol. 1993, 48(4):M167-74

19. Bø K, Talseth T, Holme I. Single, blind, randomized controlled trial of pelvicfloor exercises, electrical stimulation, vaginal cones and no treatment in management of genuine stress incontinence in women. BMJ, 1999, 318: 487-93

20. Schmitt JJ, Singh R, Weaver AL, Mara KC, Harvey-Springer RR, Fick FR, et Prospective Outcomes of a Pelvic Floor Rehabilitation Program Including A6. Vaginal Electrogalvanic Stimulation for Urinary, Defecatory, and Pelvic Pain Symptoms. Female Pelvic Med Reconstr Surg, 2017, 23(2):108-113 
21. Wang QJ, Zhao YJ, Huang LX, Zhang J, Shen W. Evaluation of the effect of electrical stimulation combined with biofeedback therapy for postpartum pelvic organ prolapse: a static and dynamic magnetic resonance imaging study [Article in Chinese] Zhonghua Yi Xue Za Zhi, 2019, 99(5): 375-379

22. Araujo CC, Coelho SSA, Martinho N, Tanaka M, Jales RM, Juliato CRT.Clinical and ultrasonographic evaluation of the pelvic floor in primiparous women: a cross-sectional study.Int Urogynecol J. 2018, 29(10): 1543-1549

23. Van Geelen H, Ostergard D, Sand P.A review of the impact of pregnancy and childbirth on pelvic floor function as assessed by objective measurement techniques. Int Urogynecol J, 2018, 29(3):327-338.

24. Bø K, Ellstrøm Engh M, Hilde G. Regular exercisers have stronger pelvic floor muscles than nonregular exercisers at midpregnancy.Am J Obstet Gynecol. 2018, 218(4): 427.e1-427.e5

25. Behnia-Willison F, Nguyen TTT, Mohamadi B, Vancaillie TG, Lam A, Willison NN, et al. Fractional $\mathrm{CO}_{2}$ laser for treatment of stress urinary incontinence. Eur J Obstet Gynecol Reprod Biol X. 2019, 11, 1:100004

\section{Tables}

Table 1. Modified Oxford scale for digital evaluation of pelvic floor muscle strength

\begin{tabular}{ll}
\hline Grade & Description \\
\hline 0 & Nil \\
1 & Flicker \\
2 & Weak \\
3 & Moderate, slight lift of the examiner's fingers, no resistance \\
4 & Good, sufficient to elevate the examiner's fingers against light resistance \\
5 & Strong, sufficient to elevate the examiner's fingers' against strong resistance \\
\hline
\end{tabular}

Table 2 Comparison of demographic data between two groups

\begin{tabular}{|c|c|c|c|c|}
\hline Variables & $\begin{array}{l}\text { PFMT Group } \\
\quad(n=69)\end{array}$ & $\begin{array}{l}\text { Control Group } \\
(\mathrm{n}=81)\end{array}$ & $t$ or $\mathrm{X}^{2}$ & $P$ value \\
\hline $\begin{array}{c}\text { Age } \\
\text { (y) }[\text { Mean } \pm S D]\end{array}$ & $28.0 \pm 2.4$ & $28.3 \pm 2.9$ & -0.666 & 0.506 \\
\hline Gestational weight gain $(\mathrm{kg})[\mathrm{Mean} \pm \mathrm{SD}]$ & $15.6 \pm 5.5$ & $17.9 \pm 8.8$ & -1.849 & 0.066 \\
\hline BMI of delivery $\left(\mathrm{kg} / \mathrm{m}^{2}\right)[$ Mean $\pm \mathrm{SD}]$ & $27.9 \pm 2.5$ & $27.4 \pm 2.6$ & 1.344 & 0.183 \\
\hline Gestational age at birth(w) [Mean \pm SD] & $39.3 \pm 1.2$ & $39.5 \pm 1.5$ & -0.900 & 0.370 \\
\hline Birth weight(g) $[$ Mean \pm SD] & $3321.7 \pm 510.6$ & $3259.3 \pm 522.1$ & 0.738 & 0.462 \\
\hline Cesarean delivery(n) & 6 & 9 & & \\
\hline Nature vaginal delivery(n) & 60 & 70 & 0.613 & 0.736 \\
\hline Instrument-assisted vaginal delivery(n) & 3 & 2 & & \\
\hline
\end{tabular}

Student's test and Chi-square test are used.

Table 3ロComparison with the PFM strength before and after treatment between the two group 


\begin{tabular}{ccc}
\hline & The number of SUI & The recovery rate of SUI (\%) \\
\hline Treatment group & 69 & $63.8 \%(44 / 69)$ \\
\hline Control group & 81 & $12.3 \%(10 / 81)$ \\
& & \\
\hline$Z$ & & 42.763 \\
\hline$P$ value & 0.000 \\
\hline
\end{tabular}

Chi-square test is used

Table 4₫Comparison of PFM strength before and after treatment between two groups

\begin{tabular}{|c|c|c|c|c|c|c|c|}
\hline \multirow[t]{2}{*}{ Variables } & \multicolumn{2}{|c|}{ Treatment $(n=69)$} & \multicolumn{2}{|c|}{$\begin{array}{l}\text { Control } \\
(\mathrm{n}=81)\end{array}$} & \multirow[t]{2}{*}{ Goups } & \multirow[t]{2}{*}{$Z$} & \multirow[t]{2}{*}{$P$ value } \\
\hline & before[ & After $\mathrm{u}$ & before! & after[ & & & \\
\hline 0 & 7 & 2 & 8 & 8 & 0-1 & -7.810 & 0.000 \\
\hline 1 & 54 & 10 & 64 & 62 & प-口 & -1.732 & 0.083 \\
\hline 2 & 6 & 50 & 5 & 6 & प-口 & -1.734 & 0.083 \\
\hline 3 & 2 & 5 & 4 & 5 & ए-प & -3.680 & 0.000 \\
\hline 4 & 0 & 2 & 0 & 0 & & & \\
\hline 5 & 0 & 0 & 0 & 0 & & & \\
\hline
\end{tabular}

Wilcoxon test is used

Table 5 Comparison of parameters related to surface electromyography(sEMG) before and after PFM training

\begin{tabular}{|c|c|c|c|c|c|c|c|}
\hline \multirow[t]{2}{*}{ Variables } & \multicolumn{2}{|c|}{ Treatment $(n=69)$} & \multicolumn{2}{|c|}{ Control $(\mathrm{n}=81)$} & \multirow[t]{2}{*}{ Goups } & \multirow[t]{2}{*}{$\mathrm{t}$} & \multirow[t]{2}{*}{$P$ value } \\
\hline & before $]$ & After $\square$ & before $\square$ & after! & & & \\
\hline $\begin{array}{c}\text { sEMG flicks } \\
(\mathrm{mV})[\text { Mean } \pm \mathrm{SD}]\end{array}$ & $11.9 \pm 4.1$ & $24.1 \pm 8.4$ & $12.0 \pm 4.2$ & $12.3 \pm 4.8$ & $\begin{array}{l}\square-\square \\
\square-\square \\
\square-\square \\
\square-\square \\
\end{array}$ & $\begin{array}{c}-14.189 \\
-1.054 \\
-0.139 \\
10.739\end{array}$ & $\begin{array}{l}0.000 \\
0.295 \\
0.890 \\
0.000\end{array}$ \\
\hline $\begin{array}{l}\text { sEMG MVC } 10 \mathrm{~s} \\
(\mathrm{mV})[\mathrm{Mean} \pm \mathrm{SD}]\end{array}$ & $16.4 \pm 5.5$ & $30.0 \pm 10.6$ & $16.2 \pm 5.5$ & $17.0 \pm 6.0$ & $\begin{array}{l}-\square \\
\square-\square \\
\square-\square \\
\square-\square \\
\end{array}$ & $\begin{array}{c}-13.058 \\
-1.981 \\
0.302 \\
9.434 \\
\end{array}$ & $\begin{array}{l}0.000 \\
0.051 \\
0.763 \\
0.000 \\
\end{array}$ \\
\hline EMG MVC $60 \mathrm{~s}(\mathrm{mV})[$ Mean \pm SD] & $15.8 \pm 5.2$ & $26.9 \pm 8.8$ & $16.3 \pm 5.2$ & $16.9 \pm 5.3$ & $\begin{array}{l}\square-[ \\
\square-\square \\
\square-\square \\
\square-\square\end{array}$ & $\begin{array}{c}-12.735 \\
-1.821 \\
-0.607 \\
8.592\end{array}$ & $\begin{array}{l}0.000 \\
0.072 \\
0.545 \\
0.000\end{array}$ \\
\hline
\end{tabular}

Student's t test is used

\section{Figures}

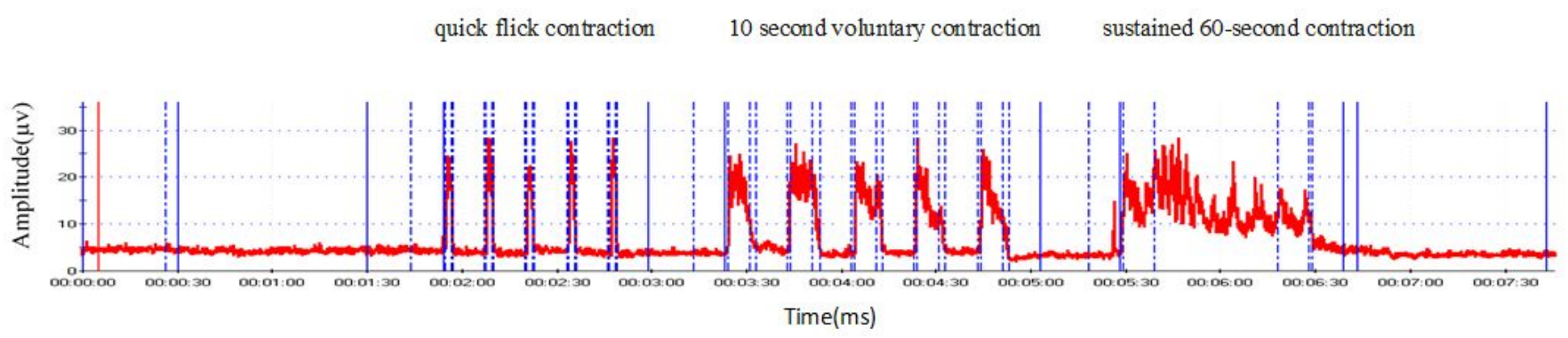

Figure 1

sEMG signal 


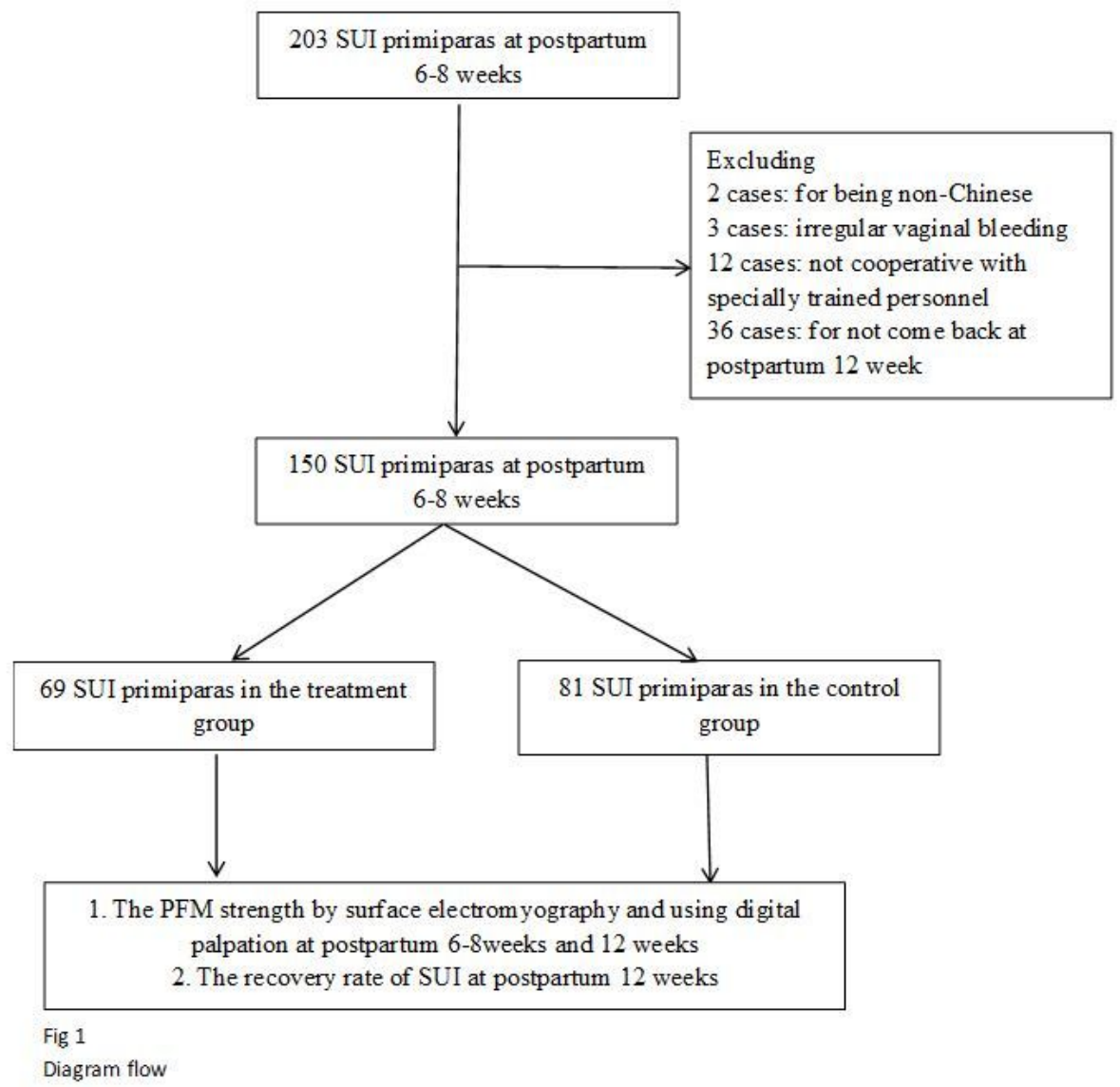

Figure 2

Flow diagram

\section{Supplementary Files}

This is a list of supplementary files associated with this preprint. Click to download.

- 20191003CONSORTchecklist.doc

- additionalfile1.xIsx 